\title{
ESTUDIO DEL ANCHO EFICAZ EN SECCIONES EN T DE HORMIGON ARMADO E IDENTIFICACION DE LOS PARAMETROS SIGNIFICATIVOS EN SU RESPUESTA ESTRUCTURAL
}

\author{
(STUDY OF THE EFFECTIVE WIDTH IN REINFORCED CONCRETE T SECTIONS AND IDENTIFICATION \\ OF SIGNIFICANT PARAMETERS IN STRUCTURAL RESPONSE)
}

\author{
Antonio R. Marí Bernat \\ Juan Miquel Canet \\ Profesores Titulares. Departamento de Estructuras \\ E.T.S. Ingenieros de Caminos de Barcelona
}

452-10

\section{RESUMEN}

En el presente trabajo se realiza un estudio del ancho eficaz de distintos tipos de secciones.

El método de análisis utilizado es el de los elementos finitos, usando elementos lámina y viga para modelizar las cabezas y almas respectivamente.

El estudio pretende identificar los parámetros básicos que influyen de forma relevante en la determinación de dicho ancho eficaz. Para ello, se ha realizado un estudio paramétrico en régimen lineal elástico, como consecuencia del cual se proponen unas expresiones sencillas. Los resultados obtenidos se han comparado con otros procedentes de soluciones analíticas (para algunos casos particulares) y experimentales, así como con los resultados obtenidos mediante aplicación de la vigente Normativa Española del Hormigón EH-82, extrayendo conclusiones al respecto.
SUMMARY

A study of the effective width of different types of reinforced concrete cross sections is presented.

The method of analysis used is the finite elements method, using shell elements and beam elements to represent the

flanges and web respectively.

One of the objectives of this study is to identify the more significant parameters in the determination of the effective width. For this purpose, a parametric study has been performed and a number of simple analytical expressions for the effective width is proposed. The obtained results have been compared with the ones coming from other analytical methods and with the ones proposed by the Spanish Concrete Code. Finally some conclusions have been derived.

\section{INTRODUCCION}

Conforme a la teoría elemental de Resistencia de Materiales, las tensiones normales en una pieza trabajando a flexión se distribuyen

proporcionalmente a la distancia a la fibra neutra de la sección, por lo que las tensiones en las alas de una viga, por ejemplo en «T», se distribuirán uniformemente. Sin embargo, si este ancho es muy grande, las partes del ala más alejadas del alma o nervio de la viga, contribuirán en menor medida a resistir el momento flector, por lo que la viga será menos resistente de lo que la teoría predice.

El hecho de que la distribución de tensiones longitudinales en las alas no sea uniforme se debe a la aparición de unas tensiones rasantes alma-ala de la viga (Fig. 1). Dichas tensiones rasantes serán tales que compatibilicen los desplazamientos

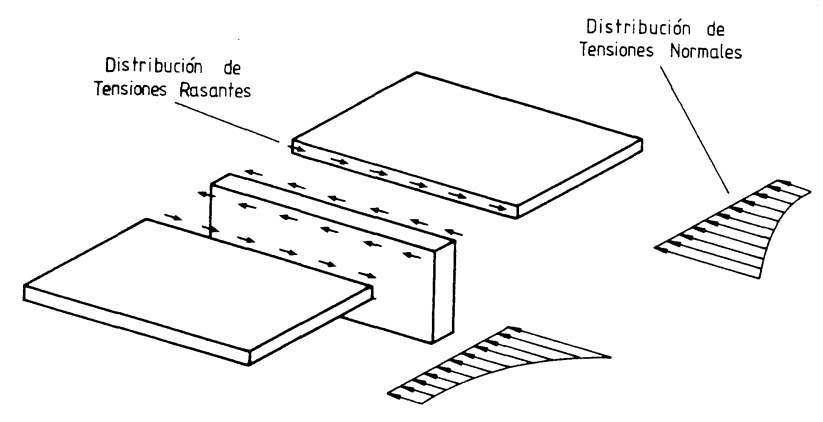

Fig. 1.-Tensiones rasantes en la unión ala-alma de la viga. 

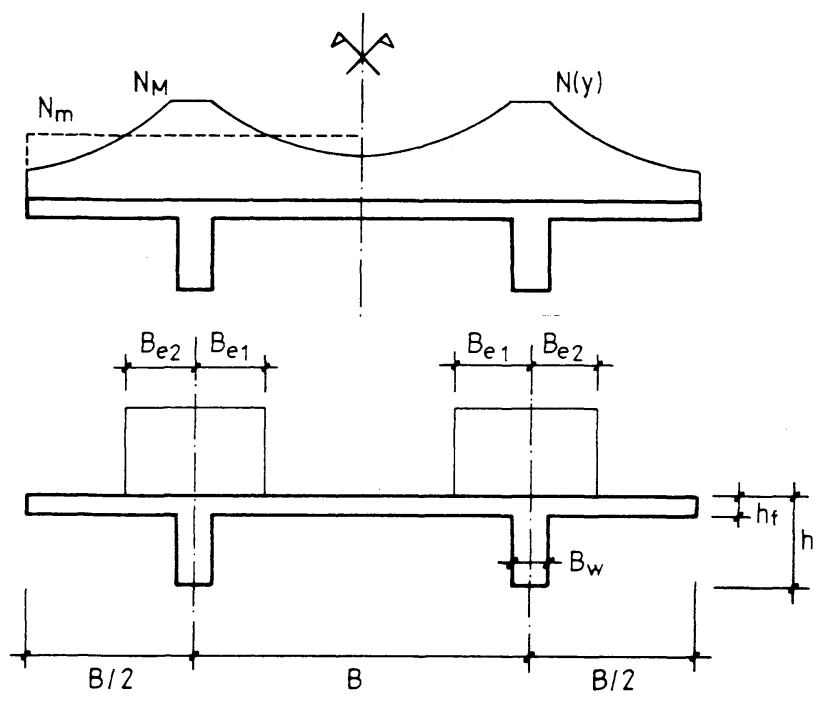

Fig. 2.-Geometria del elemento lámina.

de las alas y del alma. Considerando el ala como un elemento laja aislado sometido a esta distribución de tensiones rasantes, aplicando la teoría de la Elasticidad podemos inferir, que efectivamente la distribución de tensiones normales longitudinales no será uniforme en el ancho de la pieza.

Una manera de abordar este problema de forma práctica es suponer una anchura eficaz de ala menor que la real, de tal forma que al aplicar la teoría elemental de la flexión de vigas a la nueva sección ficticia se obtenga un valor correcto de la resistencia (Fig. 2).

La solución de este problema fue tratada primeramente por Th. von Karman (1) y posteriormente por Shnadel (2), Reissner (3), Chwalla (4) y Beschkine (5), en vigas en «T». Se supuso una viga continua, apoyada sobre un número infinito de apoyos equidistantes. El ancho del ala se suponía infinito y su espesor muy pequeño con respecto a la altura del nervio, de tal forma que se la consideraba como una laja y por tanto se la estudiaba en elasticidad bidimensional.

Recientemente, Qi-Gen Song (6), en un interesante trabajo, ha estudiado el problema del ancho eficaz, utilizando la teoría de las láminas plegadas, realizando, asimismo, un análisis de la convergencia de las series de Fourier utilizadas en dicha teoría.

En el presente trabajo, se ha intentado una aproximación al estudio de este fenómeno mediante elementos finitos, que ha permitido sacar unas primeras conclusiones cuantitativas en régimen elástico en secciones «T», «PI», cajón y «U» invertida, así como unas conclusiones de tipo cualitativo para el régimen no lineal.

\section{PROCEDIMIENTO DE CALCULO UTILIZADO EN ESTE TRABAJO}

La solución del problema del ancho eficaz, puede actualmente con la ayuda de las técnicas numéricas resolverse fundamentalmente de tres formas:

- Teoría de las láminas plegadas.

- Método de la banda finita.

- Método de los elementos finitos.

Se ha escogido la tercera alternativa, mediante un programa general de láminas, por varias razones: En primer lugar, permite también el estudio en régimen no lineal. Asimismo, se tiene en cuenta la existencia de nervios, con los cuales, como se verá más adelante, se han modelado las almas. Además, es posible también tener en cuenta la existencia de armaduras en el hormigón, las cuales si apenas modifican la respuesta en el cálculo elástico lineal, sí es de fundamental importancia el considerarlas en la alternativa no elástica $(*)$.

\subsection{Modelización de la estructura}

En la modelización de la estructura mediante elementos finitos, se han utilizado dos tipos de elementos distintos: para la lámina se ha escogido el elemento isoparamétrico degenerado de nueve nodos de la familia de Ahmad (7), con un campo de desplazamientos que se aproxima mediante funciones de interpolación lagrangianas (Fig 3).

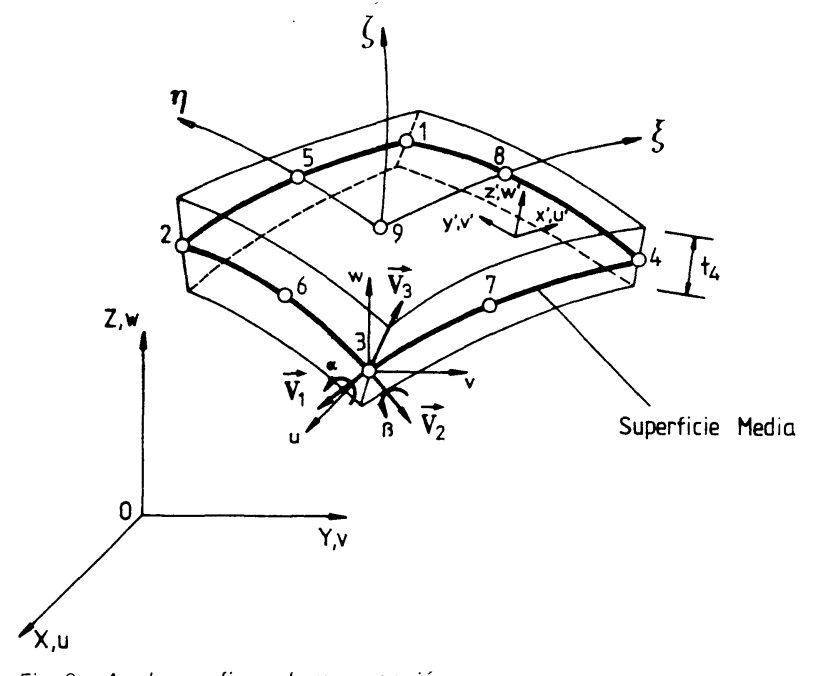

Fig. 3.-Anchura eficaz de una sección.

$\left(^{*}\right)$ Una descripción más detallada sobre la formulación del elemento finito utilizado, asi como un primer intento del estudio del ancho eficaz en régimen no lineal podrá encontrarse en un artículo de los autores que será próximamente publicado en la revista Hormigón y Acero. 


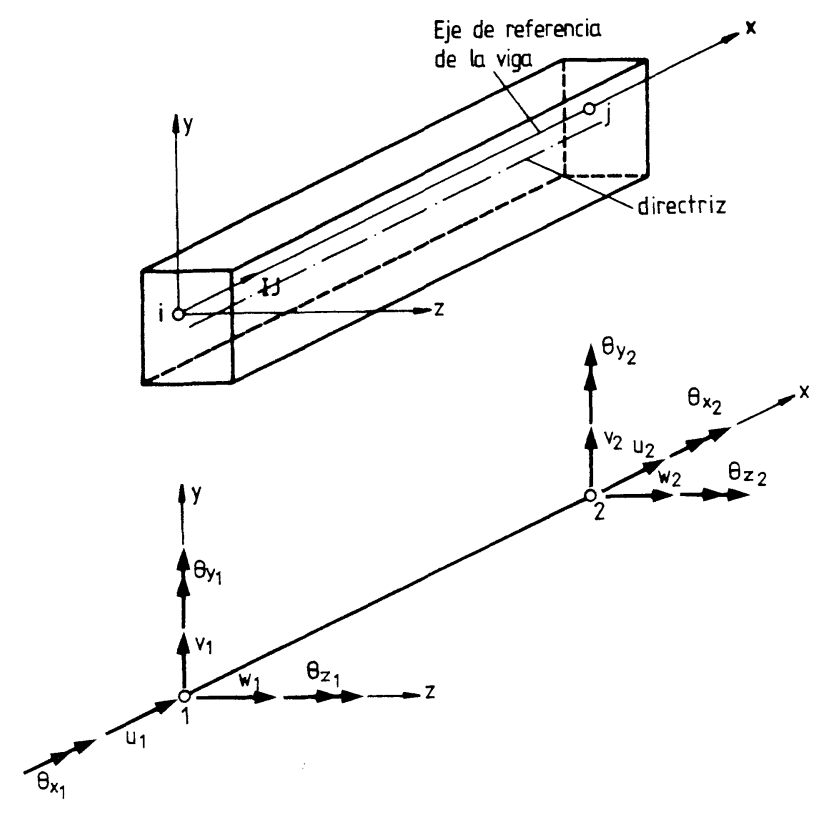

Fig. 4.-Elemento viga.

Se admite la hipótesis de que las rectas normales a la superficie media antes de la deformación, siguen rectas después de producirse ésta pero no necesariamente normales a la superficie media deformada, lo cual permite considerar, de una forma bastante aproximada, la contribución del esfuerzo cortante a la energía de deformación. Con ello, es posible el estudio de láminas gruesas. Asimismo, se hace la hipótesis habitual de que las tensiones normales a la superficie media de la lámina son despreciables.

Transversalmente, el espesor se divide en una serie de capas a fin de poder tener en cuenta la variación del estado tenso-deformacional en dicho espesor en régimen no lineal. Cada capa se supone sometida a un estado biaxil de tensiones. Asimismo, esta disposición en capas permite introducir el armado de forma muy cómoda.

En cuanto a las vigas, se discretizan mediante elementos rectos unidimensionales con dos nodos en los extremos, cada uno de ellos con seis grados de libertad. La sección transversal se divide en filamentos, en cada uno de los cuales se supone un estado tensional uniforme. De esta forma, es posible tener en cuenta la solicitación de flexión esviada sobre la viga (Fig. 4).

\section{ESTUDIO DEL ANCHO EFICAZ}

En este apartado, se entra propiamente en el análisis del fenómeno empleando los procedimientos de cálculo descritos.

El objetivo primordial es identificar los parámetros más significativos y definir su influencia en la determinación del ancho eficaz. En función de los resultados obtenidos se proponen unas fórmulas aproximadas para su uso en dimensionamiento.

\subsection{Secciones analizadas}

El primer caso que se analiza es el correspondiente a un puente recto biapoyado y sección en "PI» (Fig. 5). El armado que se le ha dispuesto (Fig 6), es el necesario para resistir, sin tener en cuenta el reparto transversal, las acciones que señala la vigente Instrucción Española de Puentes para una luz de 20 metros.

Debido a la doble simetría se ha estudiado únicamente un cuarto de pieza. La discretización

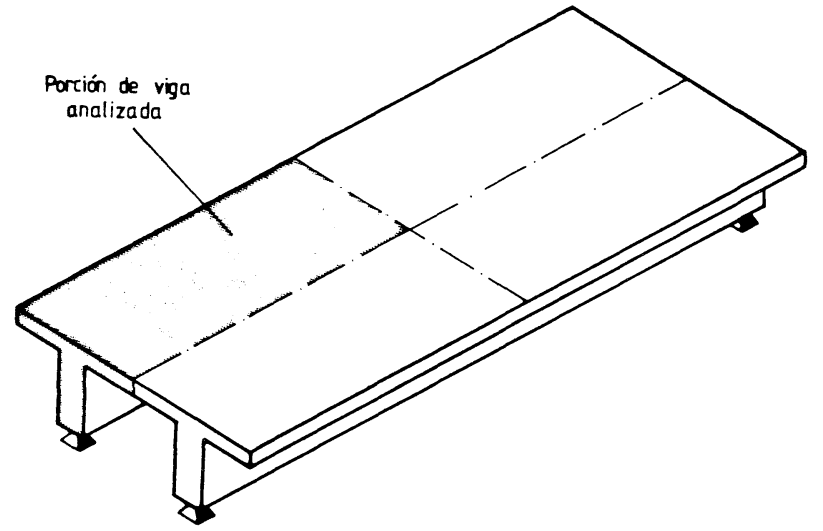

Fig. 5.-Puente con sección en PI analizado.

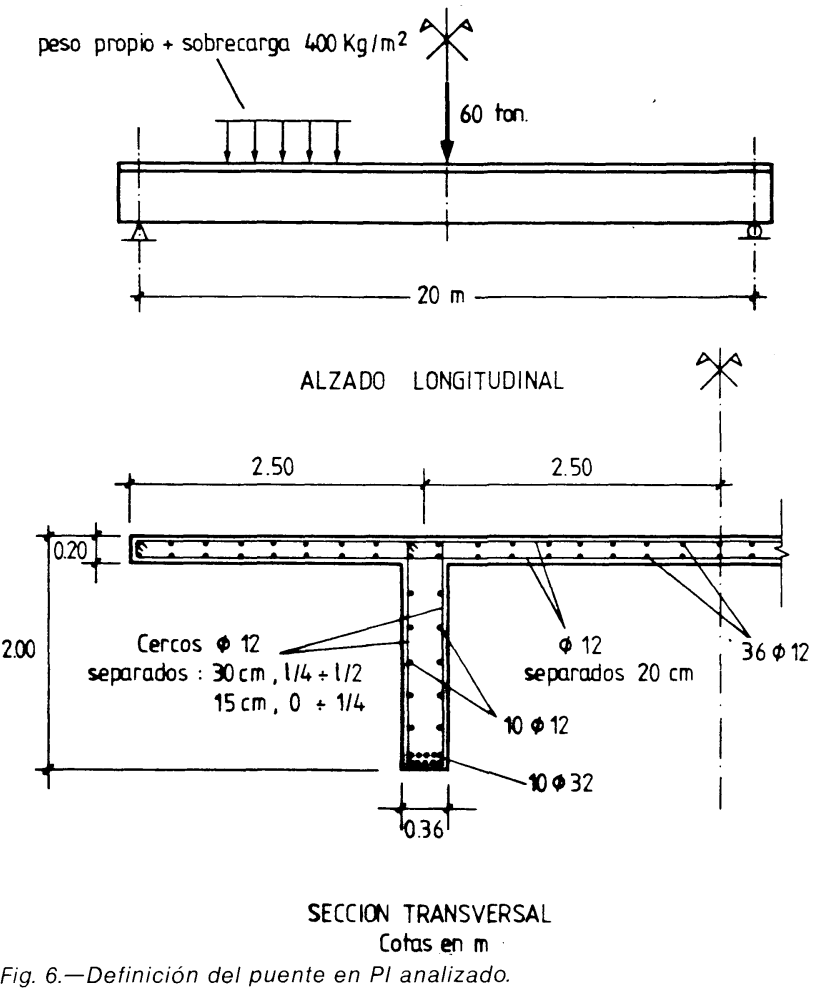




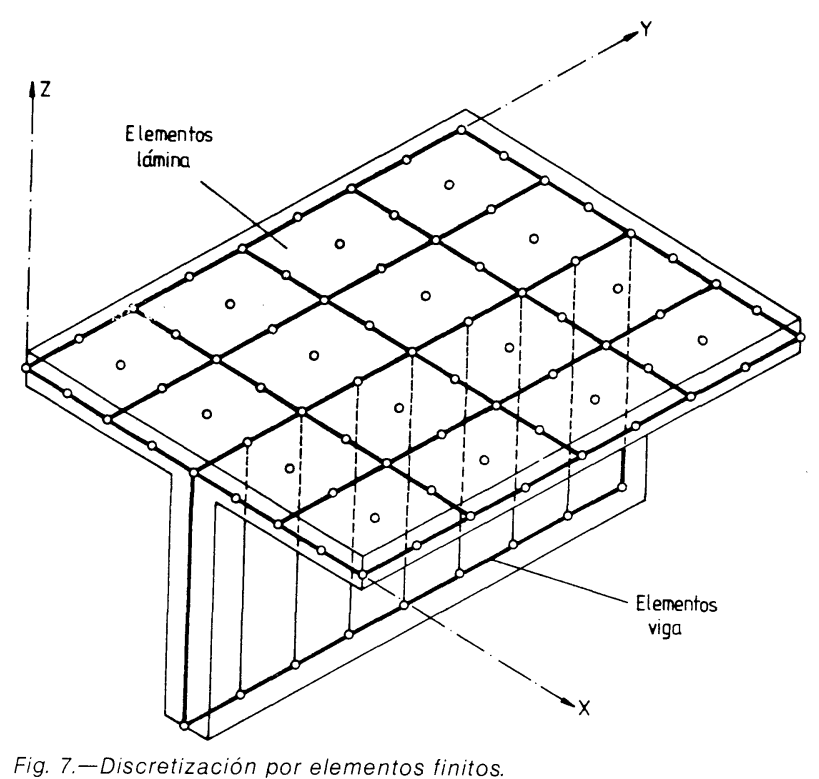

Fig. 7.-Discretización por elementos finitos.

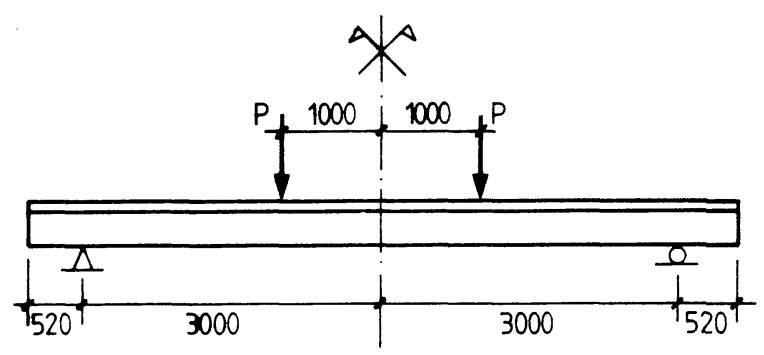

ALZADO LONGITUDINAL

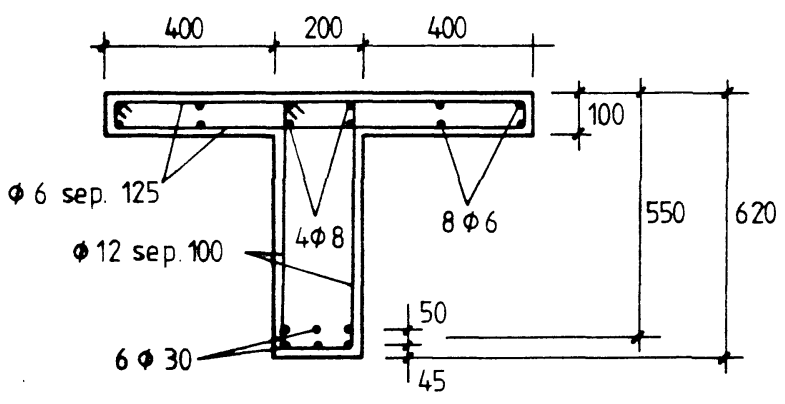

SECCION TRANSVERSAL Cotas en mm.

Fig. 8.-Viga de Razaqpur y Ghali.

por elementos finitos utilizada es la que muestra la figura 7. Como puede verse el alma se ha modelizado como una viga, ya que en los tanteos previos que se realizaron pudo observarse la similitud de resultados entre el tratamiento como viga y como lámina.

El segundo tipo de viga analizado es una sección en «T». Se han estudiado dos casos diferentes. El primero parte del esquema de viga biapoyada con sección en «PI», simplemente variando las condiciones de vinculación del borde simétrico de la «PI» y dejándolo libre. La discretización, por lo tanto, así como las propiedades de los materiales, son idénticas.

El segundo caso también corresponde a un esquema estático biapoyado. El tipo de viga es similar a la tratada por Razaqpur y Ghali (10), los cuales ensayaron hasta rotura y midieron las tensiones en los diferentes puntos.

La definición geométrica, así como la disposición de armaduras en la sección y el esquema de cargas puede verse en la figura 8.

La sección cajón analizada parte del esquema de sección en "PI» anterior, simplemente situando los nervios en los extremos del tablero y añadiendo un elemento losa inferior que cierre la sección.

Asimismo, se ha estudiado una sección en "U» invertida, idéntica a la cajón sin el tablero inferior.

\subsection{Propiedades de los materiales}

Las propiedades de los materiales son las que se indican a continuación:

Hormigón:

- Resistencia a compresión a los 28 días:

- Resistencia a tracción:

- Módulo de Elasticidad:

- Módulo de Poisson:

- Peso específico:

- Deformación para la máxima resistencia:

$300 \mathrm{kp} / \mathrm{cm}^{2}$ $50 \mathrm{kp} / \mathrm{cm}^{2}$ $350.000 \mathrm{kp} / \mathrm{cm}^{2}$ 0,15

$2,5 \mathrm{t} / \mathrm{m}^{3}$

0,002

Acero:

- Módulo de Elasticidad:

- Módulo de endurecimiento:

- Tensión de fluencia:

- Deformación última:
$2.100 .000 \mathrm{kp} / \mathrm{cm}^{2}$ $0 \mathrm{kp} / \mathrm{cm}^{2}$
$6.000 \mathrm{kp} / \mathrm{cm}^{2}$
0,1

La serie de resultados obtenidos con base a este esquema provienen de variar las proporciones geométricas de la estructura y de las distintas hipótesis de carga, a fin de determinar la influencia de los distintos parámetros que intervienen en el fenómeno.

\subsection{Parámetros estudiados}

Existen una serie de parámetros que caracterizan el ancho eficaz, los cuales son fundamentalmente de tres tipos: en primer lugar los parámetros geométricos, en segundo lugar el tipo de cargas y por último las propiedades elásticas de los materiales. 


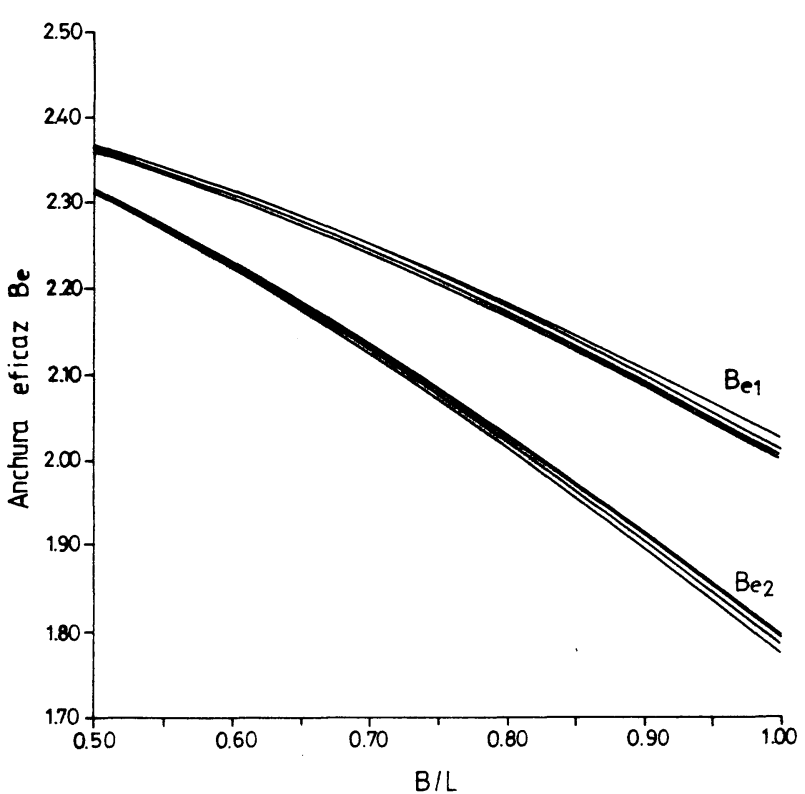

Fig. 9. - Ancho eficaz para distintos valores $h_{t} / h$.

De entre los parámetros geométricos se han estudiado:

- Relación canto de la losa/canto total.

- Relación ancho/luz.

- Anchura de los nervios/anchura de la sección.

Por lo que hace referencia a las cargas se ha estudiado el efecto en el ancho eficaz de la carga repartida y de la carga puntual.

Por último se ha analizado también la influencia del módulo de Poisson en el ancho eficaz.

\section{RESULTADOS OBTENIDOS}

\subsection{Sección en «PI»}

\subsubsection{Parámetros " $h / h$ " y " $B / B_{\omega}$ "}

«h/ $/ h »$

Manteniendo constante el valor « $\mathrm{B} / \mathrm{B}_{\omega}$ » igual a $10 / 0,36=13,8889$ se varió el espesor de la losa $\mathrm{h}=0,2, \mathrm{~h}=0,3, \mathrm{~h}=0,4, \mathrm{~h}=0,6$, los cuales dan respectivamente unas relaciones $h_{f} / h=0,1$, $h_{f} / h=0,15, h_{f} / h=0,2$ y $h_{f} / h=0,3$.

$« \mathrm{~B} / \mathrm{B}_{\omega} »:$

Manteniendo constante $h_{f} / h$ e igual a $0,20 / 2=0,1$, se varió el ancho del nervio de tal manera que se obtuvieron para $B / B_{\omega}$ los valores $\mathrm{B}^{\prime} \mathrm{B}_{\omega}=10, \mathrm{~B} / \mathrm{B}_{\omega}=5$ y $\mathrm{B} / \mathrm{B}_{\omega}=2,5$.

Cada una de estas relaciones de $h_{f} / h$ y $B / B_{\omega}$ se estudió para tres relaciones $\mathrm{B} / \mathrm{L}$ distintas,

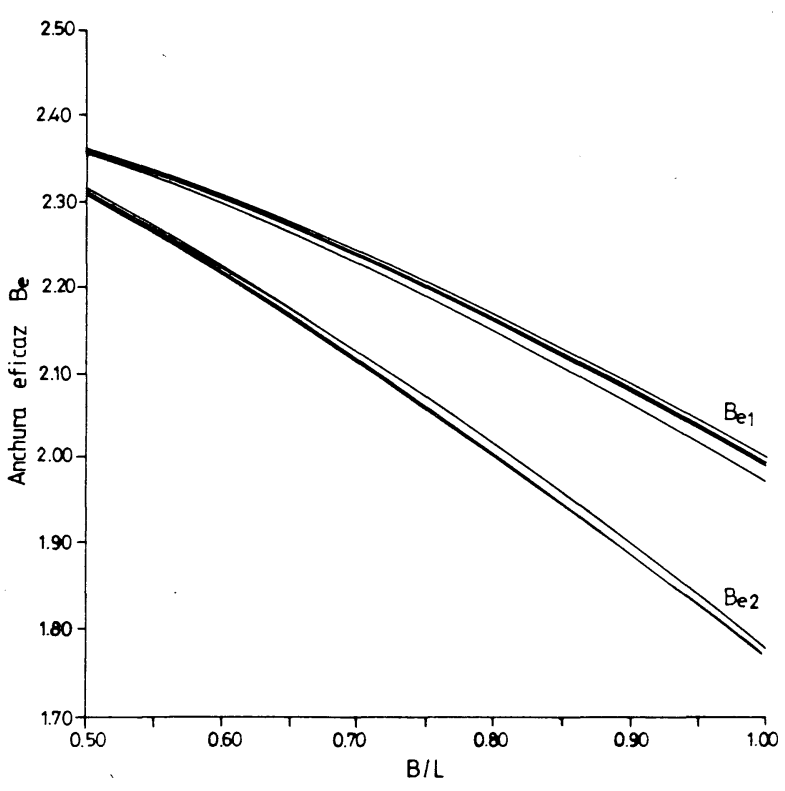

Fig. 10.-Ancho eficaz para distintos valores $B / B_{\omega}$.

correspondientes a luces de $\mathrm{L}=20 \mathrm{~m}, \mathrm{~L}=15 \mathrm{~m}$ y $L=10 \mathrm{~m}$. Los resultados obtenidos en el centro de la luz, para una carga uniformemente repartida a lo largo de la pieza de $12,24 \mathrm{t} / \mathrm{m}$, pueden verse en las figuras 9 y 10 . Como se observa en ellas, los valores del ancho eficaz son prácticamente independientes de las relaciones $h_{f} / h$ y $B / B_{\omega}$ dentro de los intervalos de variación considerados.

\subsubsection{Relación " $B / L$ ". Carga uniformemente repartida $(v=0,15)$}

Este es el parámetro geométrico más importante en el estudio del ancho eficaz, según se desprende de los resultados obtenidos.

Para analizar su influencia se han estudiado diecisiete casos distintos, partiendo de la sección en «PI» descrita en el apartado 3.1, variando la distancia entre los apoyos de la viga $\mathrm{L}=40 \mathrm{~m}$, $\mathrm{L}=24 \mathrm{~m}, \mathrm{~L}=20 \mathrm{~m}, \mathrm{~L}=17,5 \mathrm{~m}, \mathrm{~L}=15 \mathrm{~m}$, $\mathrm{L}=12,5 \mathrm{~m}$ y $\mathrm{L}=10 \mathrm{~m}$, y el ancho de la losa superior $2 \mathrm{~B}=10 \mathrm{~m}, 2 \mathrm{~B}=15 \mathrm{~m}$ y $2 \mathrm{~B}=20 \mathrm{~m}$.

Los resultados obtenidos pueden verse en la figura 11.

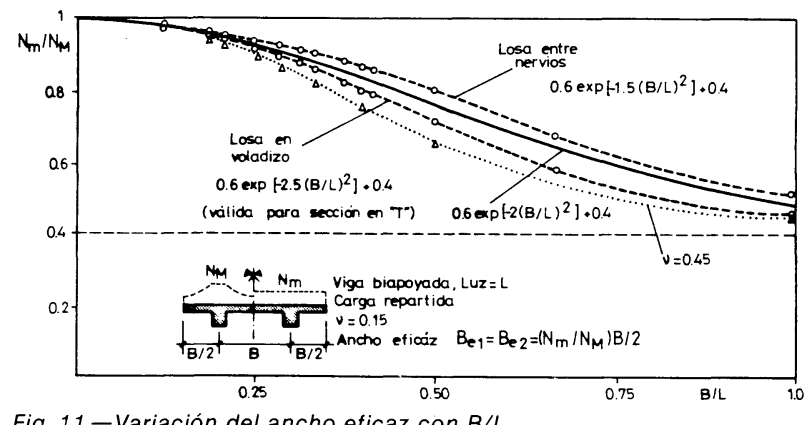

Fig. 11.-Variación del ancho eficaz con B/L 
Como puede observarse existen dos curvas, una para la losa entre nervios y otra para la losa en voladizo. Dichas curvas pueden ajustarse mediante las siguientes expresiones exponenciales:

$\sigma_{\mathrm{m}} / \sigma_{\mathrm{N}}=0,6 \exp \left[-2,5(\mathrm{~B} / \mathrm{L})^{2}\right]+0,4$ losa en voladizo $\sigma_{m} / \sigma_{M}=0,6 \exp \left[-1,5(\mathrm{~B} / \mathrm{L})^{2}\right]+0,4$ losa entre nervio por lo que el ancho eficaz vale:

$$
\mathrm{B}_{\mathrm{e}}=\frac{\sigma_{\mathrm{m}}}{\sigma_{\mathrm{M}}} \mathrm{B} / 2
$$

Estos resultados son válidos para carga uniformemente repartida y para secciones en «PI» con los nervios equidistantes del borde de la sección y del eje de simetría. No obstante, se ha comprobado que el resultado para la losa en voladizo es aplicable a secciones en «T», por lo que para este tipo de secciones se tendrá:

$\sigma_{\mathrm{m}} / \sigma_{M}=0,6 \exp \left[-2,5(\mathrm{~B} / \mathrm{L})^{2}\right]+0,4$ Sec. «T»

\subsubsection{Módulo de Poisson en relación a $B / L$}

La influencia del módulo de Poisson se estudió para distintas relaciones "B/L», dando a dicho módulo los valores $v=0,0, v=0,15, v=0,3$, $\nu=0,45$, sobre el esquema de la viga en «PI». Los resultados pueden verse en la figura 12 .

Como puede observarse, su influencia es mayor conforme aumenta la relación " $\mathrm{B} / \mathrm{L}$ », siendo muy significativa su importancia en las alas de la «PI», mientras que para la losa entre nervios dicha influencia es bastante menor. Cuando «B/L» tiende a infinito todas las curvas tienden asintóticamente a un mismo valor.

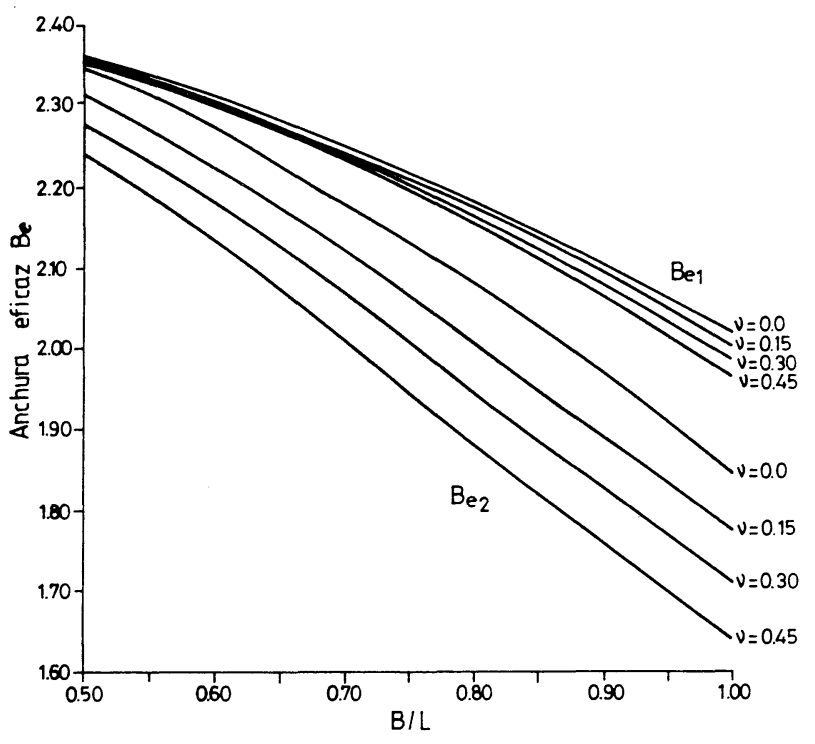

Fig. 12.-Influencia del módulo de Poisson en el ancho eficaz.

\subsubsection{Influencia del tipo de carga}

A fin de estudiar cómo influye sobre el valor del ancho eficaz el tipo de acción aplicada sobre la estructura, se analizó también la sección en «PI» bajo las siguientes hipótesis de carga:

a) Carga uniformemente repartida sobre toda la extensión del tablero, correspondiente al peso propio más la sobrecarga que especifica la Instrucción y una carga puntual de 60 toneladas, centrada transversalmente y actuando en la sección centro luz de la pieza.

b) Actuando únicamente la carga uniformemente repartida de la hipótesis a).

C) Carga puntual de 60 toneladas.

d) Carga puntual de 120 toneladas en el centro de la pieza, de tal forma que el nivel de tensiones en la sección centro-luz sea muy parecido al que se consigue para la hipótesis de carga b).

Como puede verse en las figuras 13 y 14 el ancho eficaz depende en gran medida del tipo de acción sobre la estructura. Para carga uniformemente repartida este ancho es mayor que para el caso de carga concentrada, es decir, el ancho eficaz depende de la existencia e intensidad de discontinuidades en la ley de cortantes de la viga y de la proximidad o lejanía a la sección en la cual existe tal discontinuidad. Folic (11) mostró de forma empírica que tales diferencias son más acusadas para vigas con mayor cuantía de armadura.

No se da en este trabajo ninguna expresión que cuantifique el efecto de la carga concentrada. Se ha constatado simplemente que los casos analizados se ajustan satisfactoriamente a la tabla recomendada por la Instrucción Española de Hormigón para corregir los valores del ancho eficaz correspondientes a una carga uniformemente repartida, cuando actúa una carga concentrada.

\subsubsection{Comparación con los valores recomendados por la Instrucción Española de Hormigón. Carga repartida}

A fin de hallar la posible correlación de los valores obtenidos con los recomendados por la Instrucción Española de Hormigón, se representaron dichos valores sobre las curvas obtenidas para el ancho eficaz en función del parámetro $\mathrm{B} / \mathrm{L}$, obteniéndose como resultado el que se representa en la figura 15 .

Varios comentarios pueden hacerse al respecto. En primer lugar la IEH-80 considera un parámetro $h_{f} / h$ que, tal y como se observó en el 


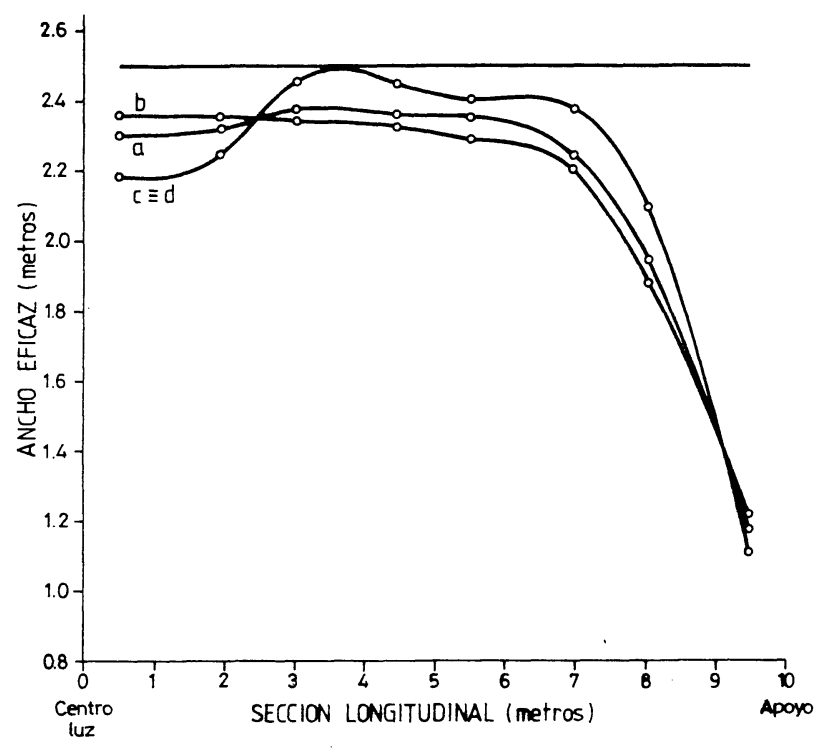

Fig. 13.-Ancho eficaz Be1.

apartado correspondiente, no tiene una influencia significativa, mientras que para la Norma dicho valor tiene gran importancia, sobre todo al crecer el parámetro $\mathrm{B} / \mathrm{L}$.

En segundo lugar, como puede apreciarse, la Norma proporciona en muchos casos valores del ancho eficaz superiores a los obtenidos en el presente estudio, sobre todo los que tienen una relación $h_{f} / h$ alta y para relaciones $B / L$ pequeñas. Análogas conclusiones pueden extraerse para el caso de secciones en «T» múltiples.

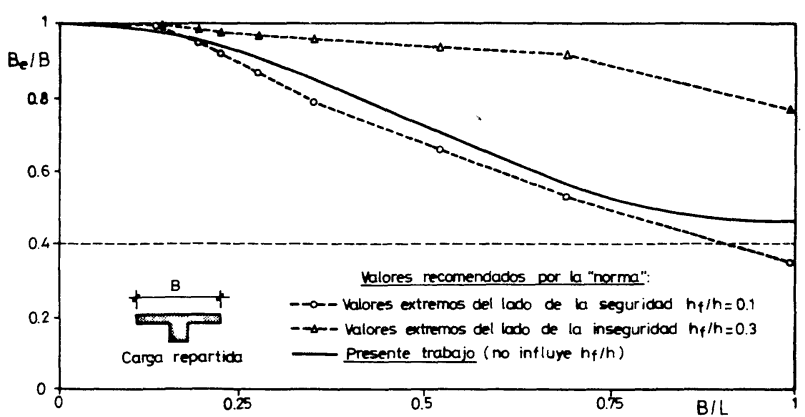

Fig. 15.-Comparación de los valores obtenidos, con los recomendados por la Instrucción Española de Hormigón.

\subsection{El ancho eficaz para secciones en «T», cajón $y$ «U» invertida, en relación con la sección en «PI»}

\subsubsection{Ancho eficaz en vigas con sección en " $T$ "}

Se considerarán en este apartado únicamente los resultados correspondientes al primer tipo de viga con sección en «T».

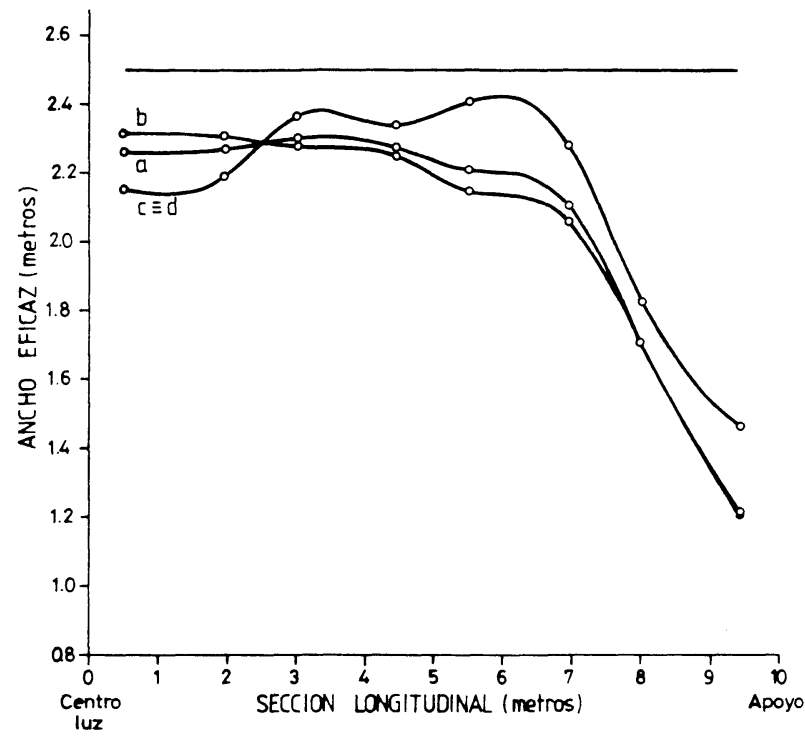

Fig. 14. - Ancho eficaz $B_{e 2}$.

Los resultados, que pueden verse en la figura 16, se adaptan muy bien a las curvas obtenidas en el apartado 4.1 para el caso de sección en «PI». Por ello pueden utilizarse para el cálculo del ancho eficaz las fórmulas exponenciales dadas en dicho apartado.

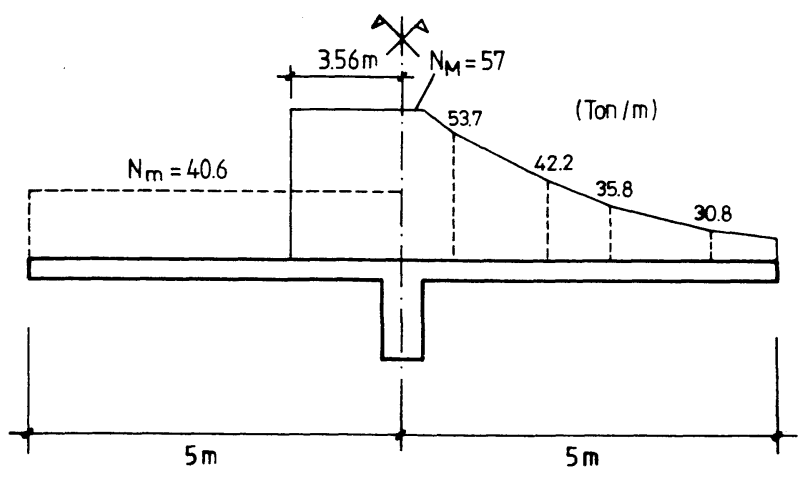

Fig. 16.-Ancho eficaz de una sección en T.

El interés en ensayar una sección de estas características, radica en la posibilidad de comparar el distinto comportamiento de las secciones en «T» y en «U» invertida que se realiza en el apartado siguiente.

\subsubsection{Ancho eficaz en secciones cajón y " $U$ " invertida}

En este apartado se estudia el ancho eficaz de un tipo de sección de uso muy frecuente en puentes: la sección cajón.

Dos propósitos se persiguen, el primero de ellos es analizar si existen diferencias significativas 


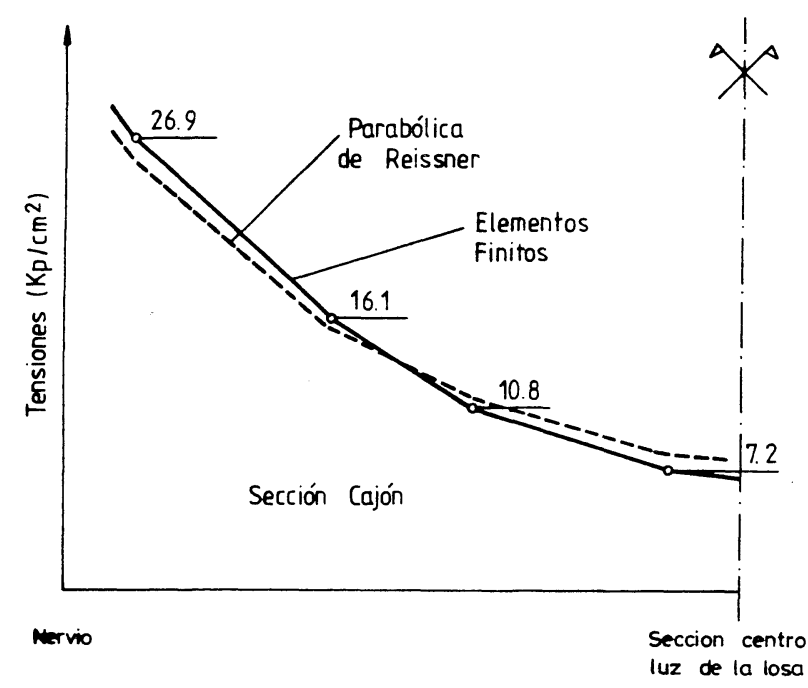

Fig. 17.-Tensiones en una sección cajón.

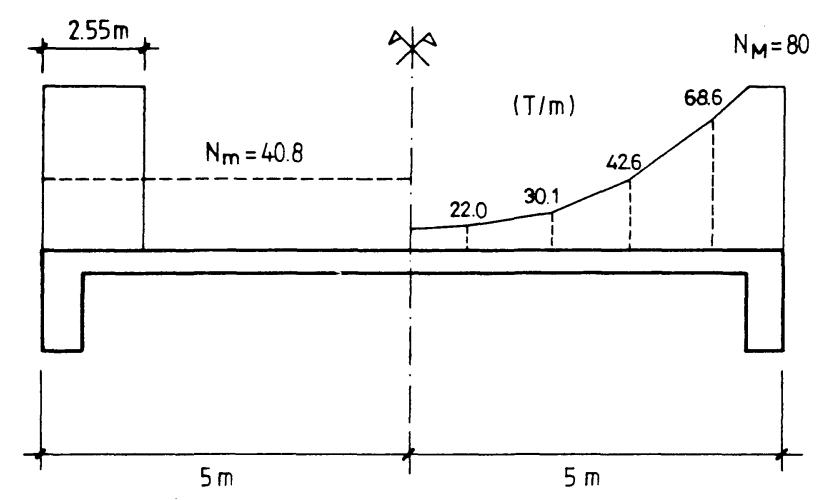

Fig. 18.-Tensiones en una "U" invertida.

respecto al ancho eficaz de la losa superior en el caso de la sección cajón (sección cerrada) y la sección abierta con iguales características geométricas, mecánicas y de carga, pero que no disponga de la losa inferior; esto es, una sección en «U» invertida.

Para ello, se han calculado dos ejemplos, por una parte un cajón rectangular y por otra una "U» invertida con idénticas características que la sección «Pl» del apartado 4.1 , pero sin la losa inferior.

Los resultados que se obtienen no presentan diferencias significativas. La distribución de tensiones en la viga con sección en " $U$ » invertida es más alta, lógicamente, por ser su inercia menor, pero la tensión máxima aumenta en la misma medida que la tensión media y el ancho eficaz es sensiblemente igual en ambos casos. Basta comparar los resultados de la viga cajón de la figura 17 con los correspondientes de la figura 18.
El segundo propósito es comparar los resultados obtenidos en este análisis con los correspondientes a otros autores:

Reissner (12), usando el principio de mínimo para la energía potencial, obtuvo unas expresiones considerando el efecto del ancho eficaz para una serie de vigas cajón rectangulares. Dichas expresiones, que parten de suponer una distribución de tensiones normales en la sección de tipo parabólico, son válidas para carga uniformemente repartida aplicada sobre las almas del cajón. Se supone, asimismo, que no existe distorsión de la sección.

Si se comparan los resultados obtenidos en el presente análisis con el correspondiente a las fórmulas de Reissner, se observa que son bastante coincidentes (Fig. 17), y que el suponer que la distribución de tensiones sigue una ley parabólica, puede dar lugar a resultados muy aceptables.

\section{VARIACION DEL ANCHO EFICAZ EN FUNCION DE LOS ANCHOS DE LA LOSA INTERMEDIA Y LA LOSA EN VOLADIZO}

En este apartado se estudian los cinco casos que se muestran en las figuras $16,18,19,20$ y 21 , representándose, asimismo, los resultados de la distribución de tensiones en cada uno de ellos. Como puede verse, para luz constante, a medida que aumenta el ancho de cada una de las partes de la losa disminuye el ancho eficaz tal como era de prever, sin embargo, no lo hace de acuerdo con las curvas exponenciales obtenidas en 4.1.3, alejándose de dichas curvas conforme nos acercamos a la sección en "U» invertida, mientras que para secciones en «T» o secciones en «Pl» con los nervios más o menos centrados, estas leyes suponen una aproximación suficiente.

Se hace hincapié en la necesidad de estudiar de una forma más exhaustiva, cómo varía el arıcho eficaz cuando cambiamos la situación de los nervios desde los casos límites de la viga en «T» y de la viga en "U» invertida.

\section{CONCLUSIONES}

A partir de los resultados obtenidos y de su comparación con los de otros autores, se puede concluir:

- El ancho eficaz depende de una serie de parámetros de los cuales los más importantes pueden clasificarse en tres tipos: geométricos, cargas sobre la estructura y módulos elásticos.

- Entre los parámetros geométricos, aparte de los referentes a la tipología considerada, el que 

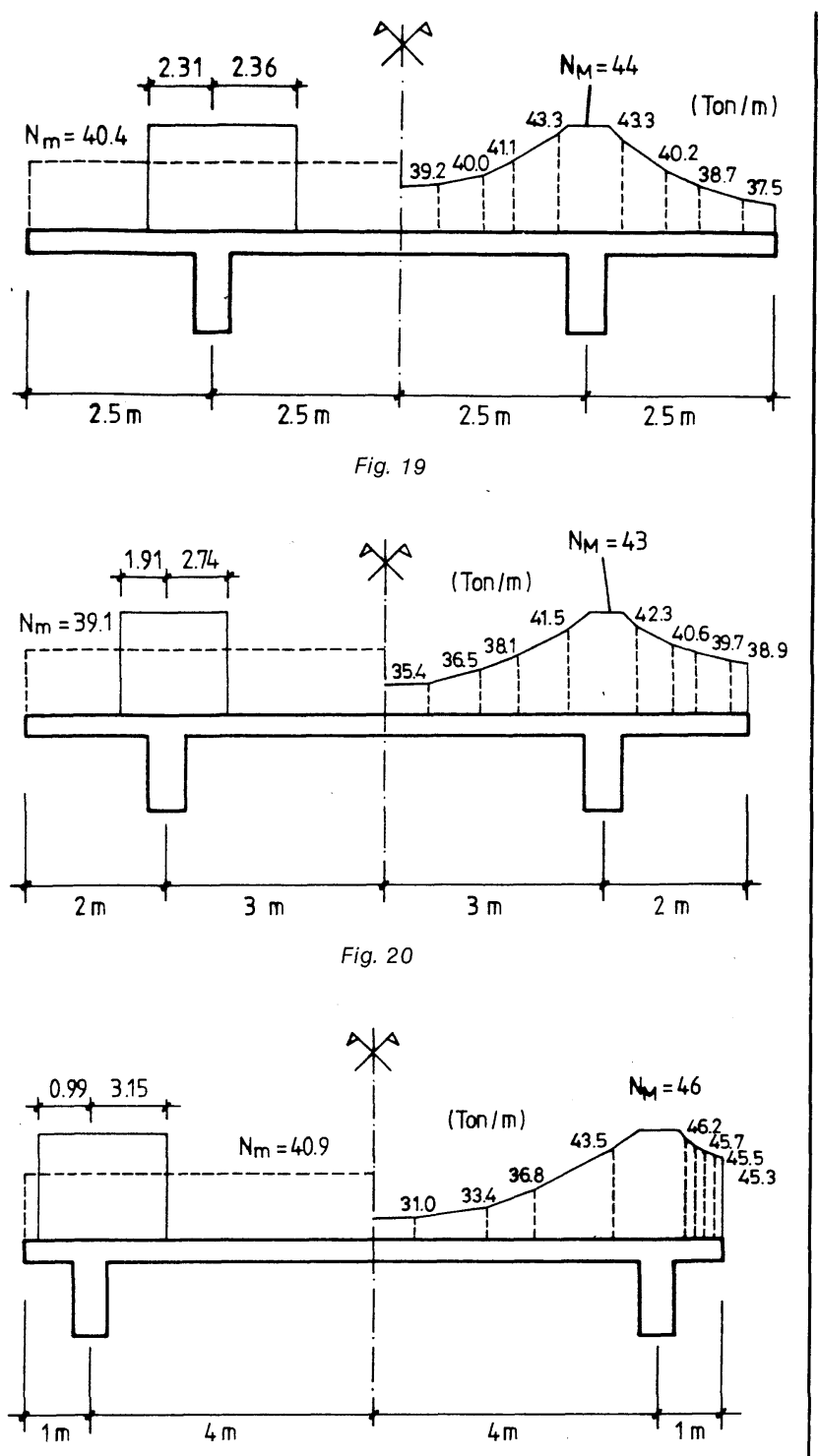

Fig. 21

se manifiesta como más significativo es la relación entre el ancho y la luz de la viga. El ancho eficaz disminuye a medida que aumenta el cociente $« B / L »$ tendiendo asintóticamente a un determinado límite.

- Otros parámetros geométricos, tales como la relación entre el espesor de la cabeza de compresión y el canto total (y por tanto de la inercia de la viga), así como la relación entre el ancho del nervio o alma y el ancho de la losa, no se han manifestado significativos en los rangos de variación normales en este tipo de estructuras.

- El tipo de cargas influye notablemente en el fenómeno, siendo esta influencia más acusada para cargas concentradas que para cargas repartidas. Asimismo, el ancho eficaz en la sección considerada depende de la distancia de dicha sección al punto de aplicación de las cargas. La distribución transversal de dichas cargas, tal como ha demostrado Folic (11) no influye.

- El módulo de Poisson es también un parámetro importante.

- Los resultados obtenidos difieren, en algunos casos, de los valores recomendados por la Instrucción Española de Hormigón. Las discrepancias más significativas son las siguientes: Los resultados de la Norma varían con el parámetro « $h_{f} / h »$ cuando en el presente trabajo se muestra que el ancho eficaz no depende de dicho parámetro. En segundo lugar, muchos de los valores dados por la Norma dan, bajo las hipótesis lineales, anchos eficaces mayores que los obtenidos en este trabajo.

\section{APENDICE}

Notación

L Luz de la viga biapoyada.

h Canto de la sección (Fig. 2).

$h_{f} \quad$ Espesor de la cabeza de compresión o losa superior (Fig. 2).

B Ancho de la sección (Fig. 2).

$B_{\omega} \quad$ Ancho del alma o nervio (Fig. 2).

$B_{\text {e }} \quad$ Ancho eficaz (Fig. 2).

$\mathrm{B}_{\mathrm{e} 1} \quad$ Ancho eficaz correspondiente a la losa entre nervios (Fig. 2).

$\mathrm{B}_{\mathrm{e} 2}$ Ancho eficaz correspondiente a la losa en voladizo (Fig. 2).

$\mathrm{N}_{M} \quad$ Esfuerzo axil máximo por unidad de longitud en la cabeza de compresión.

$\mathrm{N}_{\mathrm{m}} \quad$ Esfuerzo axil medio por unidad de longitud en la cabeza de compresión.

\section{REFERENCIAS}

1. TH. VON KARMAN: «Festschrift August Foppls». Pág. 114, 1923.

2. G. SHNADEL: «Werft und Rederei». Vol. 9, pág. 92, 1928.

3. E. REISSNER: «Der Stahlbau». Pág. 206, 1934.

4. E. CHWALLA: «Der Stahlbau». 1936.

5. L. BESCHKINE: «Publs. Int. Assoc. Bridge ans Structural Engineering». Vol. 5, pág. 65, 1938.

6. QI-GEN SONG: "Shear lag analysis of simple and continuous T, I and box beams». Report No. UCB/SESM-84/10. Department of Civil Engineering. University of California. Berkeley, California.

7. S. AHMAD: "Curved Finite Element in the Analysis of Solid, Shell and plates Structures». Ph. D. Thesis. University of Wales, Swansea, 1969

8. W. F. CHEN: "Plasticity in Reinforced Concrete». McGraw Hill, 1982.

9. «State of the Art Report on Finite Element Analysis of Reinforced Concrete». ASCE, Special Publication, 1982.

10. A. G. RAZAQPUR, and A. GHALI: "Shear Lag Analysis in Reinforced Concrete». Avanced Mechanics of Reinforced Concrete. IABSE Colloquium Delft, 1981.

11. J. FOLIC: "Analysis of the Effective Slab Width in Reinforced and Prestressed Concrete Elements». Proceedings of the International Conference on Computer Aided Analysis and Design of Concrete Structures. Pineridge Press, Swansea, 1984.

12. E. Reissner: «Analysis of the Shear Lag in Box Beams by the Principle of Minimum potential Energy». Quaterly of Applied Mathematics, October, 1946. 